\title{
THE ASSOCIATION OF HLA WITH DISEASES IN JAPANESE
}

\author{
Setsuya NarTo \\ Second Department of Internal Medicine, Fukuoka University \\ Medical School, Fukuoka 814-01, Japan
}

\begin{abstract}
Summary The diseases associated with HLA in Japanese, for which more than two laboratories have confirmed the association, were listed. The association first reported by Japanese researchers and confirmed by researchers of other countries like the association of narcolepsy and HLADR2 was stressed. There are some diseases like Takayasu' disease, the association of which was found and confirmed only in Japanese. On the other hand, there are many diseases, the association of which has been reported in the races other than Japanese. Extremely close association of HLA-B27 and ankylosing spondilitis has been observed in Japanese as well as the other races, but as to the other B27 related diseases the association is not clear in Japanese. HLA antigens associated with type I diabetes mellitus in Japanese are HLA-Bw54, DR4.1 and DwYT, which are quite different from the ones in Caucasians. The haplotype comparable to Caucasian A1-B8-DR3-Dw3 has not been found in Japanese. The mechanisms of the associations are still remain to be resolved. Some influential hypotheses such as the presence of Ir and Is genes linked to HLA loci and a single mutation were discussed.
\end{abstract}

\section{Introduction}

Intensive studies on the association of HLA with various diseases have been done throughout the world during over a past decade and the most of the association have been established. Now many researchers on this field have concentrated their efforts to find out causes of the association. There have been a lot of excellent review articles on the association of HLA with diseases outside Japan (Tiwari and Terasaki, 1985, Dausset and Svejgaard, 1977, Ryder et al., 1979, Svejgaard et al., 1983). Wakizaka (1982) reviewed the reports on HLA and diseases in Japanese very beautifully but the review article was written in Japanese. There have been no objectively reviewed articles on HLA distribution in Japanese patients with

Received October 8, 1986; Accepted November 19, 1986 
various diseases which were in English. Since phenotypic frequencies of HLA antigens are quite different among various ethnic groups and since linkage disequilibrium among HLA loci are also different in different ethnic groups, quite a few diseases have been observed to be associated with different HLA antigens among the different ethnic groups. So, some misunderstandings on the issue of HLA and diseases exist among the poeple who are not so familiar with HLA. The author intends to clarify the Japanese situation as to the diseases and HLA and compare that with the situation in other races, especially in Caucasians. However, there will be no intension to list up all the reports on this issue published in Japan in the present article. Rather, specific characters of the association of HLA with diseases in Japanese patients will be described as clearly as possible.

\section{Original contribution}

Table 1 shows diseases associated with HLA which have been reported from two or more laboratories with consistent results. Disease listed in Table 2 are

Table 1. Diseases associated definitely with HLA both in Japanese and Caucasians.

\begin{tabular}{lcc}
\hline \multicolumn{1}{c}{ Disease } & Japanese & Caucasians \\
\hline Narcolepsy & DR2 & DR2 \\
Ankylosing spondilitis & B27 & B27 \\
Diabetes mellitus type I & DR4 and DR3 & DR4 and DR3 \\
Rheumatoid arthritis & DR4 & DR4 \\
Behcet's disease & B51 & B51(Turky, Arab) \\
Psoriasis vulgaris & Cw6, B13, 17, 37, DR7 & Cw6, B13, 17, 37, DR7 \\
Pemphigus vulgaris & DR4 & DR4 (Jews) \\
Membranous glomerulopathy & DR2 & DR3 \\
SLE & DR2? & DR3 \\
Graves' disease & B35 & DR3 \\
Ulcerative colitis & Bw52 & B27 \\
Lipoid nephrosis & DQw3 & DR7
\end{tabular}

Table 2. Diseases associated with HLA in Japanese but not confirmed in Caucasians.

\begin{tabular}{lc}
\multicolumn{1}{c}{ Disease } & HLA antigen \\
\hline $\begin{array}{l}\text { Takayasu disease } \\
\text { Vogt-Koyanagi-Harada syndrom } \\
\text { Mixed connective tissue disease }\end{array}$ & Bw52 \\
& BwWa \\
\hline
\end{tabular}


recognized for the association with HLA only in Japanese patients. One hundred percent existence of HLA-DR2 in the patients with narcolepsy was originally discovered by Juji et al. (1984). This was also true in Caucasians (Langdon et al., 1984), and has become one of the most exciting topics in this fields. The auther's group first reported the significant association of HLA-Bw52 with Takayasu's disease (Naito, et al., 1978), and it was confirmed by others (Isohisa, et al., 1978; Moriuchi, et al., 1982). This association, however, has only been compatible with Japanese and yet any relation between Takayasu' disease and HLA has not been found in other races. The third original discovery of the association by Japanese was the one about Vogt-Koyanagi-Harada syndrome with HLA-Bw54,-DR4, and -DwWa, which was reported by Prof. Aizawa's group of Hokkaido University (Yakura, et al., 1976; Ohno, 1980). The relation between the syndrome and HLA in other races is also uncertain. The association of mixed connective tissue disease with HLA was reported only from Japan but it has not been confirmed yet (Sasazuki et al., 1979).

Diseases which associate with common HLA antigens between Japanese and other ethnic groups

The extremely high correlation between HLA-B27 and ankylosing spondilitis was also observed in Japanese just as same as the other races tested so far (Tiwari and Terasaki, 1985). As shown in Table 3, however, there are many diseases which are associated with HLA in Caucasians but have not been recognized for the association with HLA in Japanese. Diseases such as Reiter's syndrome, acute anterior uveitis, juvenile rheumatoid arthritis, psoriatic arthritis, and Yersinia arthritis which

Table 3. Diseases associated with HLA in Caucasians but not confirmed in Japanese.

\begin{tabular}{lc}
\multicolumn{1}{c}{ Disease } & HLA antigen \\
\hline $\begin{array}{l}\text { Multiple sclerosis } \\
\text { Goodpasture's syndrome }\end{array}$ & DR2 \\
Myasthenia gravis & DR2 \\
Chronic active hepatitis & DR3 \\
Sjoegren syndrome & DR3 \\
IgA deficiency & DR3 \\
Celiac disease & DR3 \\
Acute anterior uveitis & DR3 \\
Juvenile rheumatoid arthritis & B27 \\
Congenital adrena! hyperplasia & B27 \\
Idiopathic hemochromatosis & Bw47 \\
Hodgkin's disease & A3 \\
\hline
\end{tabular}


are also associated with HLA-B27 in Caucasian have not been reported in Japanese (Table 3).

Psoriasis vulgaris was reported to be primarily associated with HLA-Cw6 in Japanese as well as Caucasian (Tiwari and Terasaki, 1985). Recently, however, discrepant findings have been reported in- and outside Japan (Kimura et al., 1986; Laurentaci et al., 1982).

Behcet's disease is associated with HLA-Bw51 both in Japanese and Caucasians. Ulcerative colitis is associated with HLA-Bw52 in Japanese and with B27 in Caucasians (Tiwari and Terasaki, 1985). Primary association of HLA-B locus antigens with diseases except HLA-B27 is considered to be unique, because most of diseases have recently observed to be associated primarily with D-DR locus antigens.

HLA-DR4 is one of the most interesting antigens in the matter of disease association, although it has not yet been clearly defined serologically, and might be divided into several subtypes. DR4 has been reported to be associated with Pemphigus vulgaris, rheumatoid arthritis and IgA nephropathy both in Japanese and Caucasian (Tiwari and Terasaki, 1985).

\section{Diseases which associate different antigens between Japanese and Caucasians}

It has been said that one of the most important discoveries in the studies of diabetes mellitus (DM) in the last decade is the discovery of association of HLA with insulin dependent DM (IDDM) or Type I diabetes mellitus. In Caucasians, its association with HLA-A1, -B8 and/or -B15 was first reported, and much more close relation with HLA-DR3 and/or -DR4 was found later, after the discovery of D-DR loci (Tiwari and Terasaki, 1985). In Japanese, HLA-Bw54, one of the specific antigen for Japanese, was found to be associated with IDDM (Wakizaka et al., 1976; Kawa et al., 1978). Later it was discovered that Japanese IDDM was also associated with DR3 and DR4 (Sakurami et al., 1982; Batchelor and Morris, 1977). This DR4, however, might be different from the DR4 associated with Caucasian IDDM, because their association to D locus are different from each other: Japanese DR4 is associated with DwYT, and Caucasian DR4 with Dw4. The association of DR locus antigens with IDDM is a unique one, because both DR4 and DR3 have positive correlation with IDDM, but person heterozygous both DR4 and DR3 is most susceptible to IDDM. Persons with HLA-DR2 are resistant to IDDM in Japanese and Caucasians.

Many of the so called autoimmune diseases such as systemic lupus erythematosus (SLE), myasthenia gravis, Graves" disease, and active chronic hepatitis are associated with HLA-A1, -B8 and -DR3 in Caucasians. It is considered that primary associated antigen with such diseases is HLA-DR3, the association of Al and B8 being due to linkage disequilibrium. In Caucasians A1-B8-DR3-Dw3 is the most frequent haplotype with the highest linkage disequilibrium. The equivalent to such a haplotype has not been found in Japanese. There seem to be several antigens which are associated with Japanese SLE, for examples, DR2 (Hashimoto 
et al., 1985), A11 and B40 (Wakizaka, 1982). But the findings are not consistent.

The association of myasthenia gravis and HLA is still a controversial matter in Japan. The association of HLA-Bw35 with Graves' disease have been reported (Tiwari and Terasaki, 1985), but the author's group has recently discovered the association of Bw46 with Graves' disease (Naito et al., 1987) and its counterpart of DR antigen has not been clarified. It was reported that chronic active hepatitis was associated with Bw54, DR4 and Dw15 in Japanese (Ohkubo, 1979), but it has not been confirmed yet.

\section{Mechanisms of HLA and disease association}

It is now clear that the susceptibility genes for congenital adrenal hyperplasia, idiopathic hemochromatosis and olivopontcerebellar ataxia (Menzel type) are mapped in the HLA region on chromosome 6 (Robinson and Lamm, 1984). Many other diseases discussed in the present paper, however, have not been clarified on their causes of association with HLA. Various hypothesis have been proposed but thus far none is suported by clear-cut evidence.

There are two main hypothesis for basic mechanisms of HLA and disease association and the two seem to be true according to respective diseases. One is that HLA antigen itself has the role to cause disease. The representatives of the diseases caused by this mechanism might be so called HLA-B27 related diseases like ankylosing spondilitis, Reiter's disease, and acute anterior uveitis.

Many other diseases, especially diseases associated with different HLA alloantigens among different ethnic groups, will be never caused by HLA antigens themselves, but will be associated with genes linked very closely to HLA locus. The associations of the diseases and HLA will show up due to the linkage disequilibrium. This hypothesis has long been accepted by many investigators since 1974 (McDevitt and Bodmer, 1974). They postulated the Ir genes located in the HLA-D-DR gene region would be responsible for the development of diseases. Sasazuki et al. (1983) reported that Is genes existed in the HLA class II gene area might also be important to cause some diseases.

A single mutation hypothesis for the mechanism of the association between HLA and multiple sclerosis is interesting (Terasaki and Mickey, 1976). The incidence of some diseases in some ethnic groups seems to be correspondent to the frequencies of some specific HLA antigens in the ethnic groups like multiple sclerosis and HLA A3-B7-DR2-Dw2 in North Europe Caucasian, and Takayasu's disease and HLA A24-Bw52-DR2-Dw12 in Japanese. The former is common in North European and rare in Japanese. On the other hand, the latter is common in Japanese and rare in Caucasians. The relation also seems to be true for acute anterior uveitis and B27 in Caucasican, and Vogt-Koyanagi-Harada syndrome and B54-DR4-DwWa in Japanese. 


\section{REFERENCES}

Batchelor, J.R. and Morris, P.J. 1977. Juvenile onset diabetes. Joint report. In Histocompatibility Testing. Bodmer, W.F., Batchelor, J.R., Bodmer, J.G., Festenstein, H., and Morris, P.J., eds., Munksgaard, Copenhagen, pp. 211-213.

Dausset, J. and Svejgaard, A., eds. 1977. HLA and Disease. Williams \& Wilkins, Baltimore.

Hashimoto, H., Tsuda, H., Matsumoto, T., Nasu, H., Takasaki, Y., Shokawa, Y., Hirose, S., Terasaki, P.I., and Iwaki, Y. 1985. HLA antigens associated with systemic lupus erythematosus in Japan. J. Rheumatol. 12: 919-923.

Isohisa, I., Numano, F., Maezawa, H., and Sasazuki, T. 1978. HLA-Bw52 in Takayasu Disease. Tissue Antigens 12: 246-248.

Juji, T., Satake, M., Honda, Y., and Doi, Y. 1984. HLA antigens in Japanese patients with narcolepsy. All patients were DR2 positive. Tissue Antigens 24: 316-319.

Kawa, A., Nakazawa, M., Kono, Y., Sakaguchi, S., Nakamura, S., and Kanehisa, T. 1978. HLA Bw54 and B5 in Japanese diabetics with juvenile-onset and insulin-dependency (with special reference to the family history). Experimentia 34: 669-670.

Kimura, H., Naito, S., Nomoto, K., and Nakamizo, Y, 1986. Association between HLA and psoriasis in Japan. J. Dermatol. (Tokyo) in press.

Langdon, N., Welsh, K., van Dan, M., Vaughan, R.W., and Parkes, D. 1984. Genetic markers in narcolepsy, Lancet ii: 1178-1180.

Laurentaci, G., Lomuto, M., and Favoino, B. 1982. Immunogenetic analysis of association between HLA antigens and psoriasis vulgaris: Population and family studies. Dermatologica 165: 591-600.

McDevitt, H.O. and Bodmer, W.F. 1974. HL-A, immune response genes, and diseases. Lancet i: $1269-1275$.

Moriuchi, J., Wakizaka, A., Aizawa, M., Yasuda, K., Yokota, A., Tanabe, T., and Itakura, K. 1982. HLA-linked susceptibility of Takayasu disease. Hum. Immunol. 4: 87-91.

Naito, S., Arakawa, K., Saito, S., Toyoda, K., and Takeshita, A. 1978. Takayasu's disease: Association with HLA-B5. Tissue Antigens 12: 143-145.

Naito, S., Sasaki, H., and Arakawa, K. 1987. Japanese Graves' disease: Association with HLA. Bw46. submitted for publication.

Ohkubo, A. 1979. Hepatitis and HLA. J. Jpn. Soc. Intern. Med. 68: 1406-1410.

Ohno, S. 1980. Immunogenetic studies on various ocular disease. Nippon Ganka Gakkai Zasshi (in Japanese) 83: 1875-1908.

Robinson, E.B. and Lamm, L.U. 1984. Human gene mapping 7: Report of the committee on the genetic constitution of chromosome 6. Cytogenet. Cell Genet. 37: 47-70.

Ryder, L.P., Anderden, E., and Svejgaard, A. 1979. HLA and Disease Registry. Third Reports. Munksgaard, Copenhagen.

Sakurami, T., Ueno, Y., Nagaoka, K., Kuno, S., Iwaki, Y., Park, M.S., and Terasaki, P.I. 1982. HLA-DR specificities in Japanese with juvenile-onset insulin-dependent diabetes mellitus. Diabetes 31: 105-106.

Sasazuki, T., Kaneoka, H., Ohta, N., Hayase, R., and Iwamoto, I. 1979. Common HLA haplotype and their association with diseases in the Japanese population. Transplant. Proc. 11: 18711873.

Sasazuki, T., Nishimura, Y., Muto, M., and Ohta, N. 1983. HLA-linked genes controlling immune response and disease susceptibility, Immunological Rev. 70: 51-75.

Svejgaard, A., Platz, P., and Ryder, L. 1983. HLA and disease 1982-A survey. Immunological Rev. 70: 193-218.

Terasaki, P.I. and Mickey, M.R. 1976. A single mutation hypothesis for multiple sclerosis based on the HL-A system. Neurology 26: 56-58. 
Tiwari, J.L. and Terasaki, P.I. 1985. HLA and Disease Association. Springer-Verlag, New York. Wakizaka, A. 1982. Present status of intractable disease and HLA in Japan. Clin. Immunol. (in Japanese) 14 (suppl. 4): 2-16.

Wakizaka, A., Aizawa, M., Matsuura, N., Nakagawa, S., Nakayama, E., Okuno, A., and Wagatsuma, Y. 1976. HL-A and juvenile diabetes mellitus in Japanese. Lancet ii: 970.

Yakura, H., Wakizaka. A., Aizawa, M., Itakura, K., Tagawa, Y., and Sugiura, S. 1976. HLA-D antigen of Japanese origin (LD-WA) and its associating with Vogt-Koyanagi-Harada syndrome. Tissue Antigens 8: 35-42. 